Article

\title{
Cigarette Smoking Promotes Infection of Cervical Cells by High-Risk Human Papillomaviruses, but not Subsequent E7 Oncoprotein Expression
}

\author{
Kimon Chatzistamatiou ${ }^{1, *(1)}$, Theodoros Moysiadis ${ }^{2}$ (1), Dimos Vryzas ${ }^{3}$, Ekaterini Chatzaki ${ }^{4}$, \\ Andreas M. Kaufmann ${ }^{5}$ (D) , Isabel Koch ${ }^{6}$, Erwin Soutschek ${ }^{6}$, Oliver Boecher ${ }^{6}$, Athena Tsertanidou ${ }^{7}$, \\ Nikolaos Maglaveras ${ }^{8}$, Pidder Jansen-Duerr ${ }^{9}$ and Theodoros Agorastos ${ }^{7}$ \\ 1 2nd Department of Obstetrics and Gynecology, Aristotle University of Thessaloniki, \\ Hippokratio General Hospital, 54642 Thessaloniki, Greece \\ 2 Institute of Applied Biosciences, Centre for Research \& Technology-Hellas, \\ 57001 Thessaloniki, Greece; moysiadis.theodoros@gmail.com \\ 3 Medical School, Democritus University of Thrace, 68100 Alexandroupolis, Greece; d.vrizas@gmail.com \\ 4 Laboratory of Pharmacology, Medical School, Democritus University of Thrace, \\ 68100 Alexandroupolis, Greece; achatzak@med.duth.gr \\ 5 Department of Gynecology, Charité-Universitaetsmedizin Berlin, Campus Benjamin Franklin, \\ 10117 Berlin, Germany; andreas.kaufmann@charite.de \\ 6 Mikrogen GmbH, 82061 Neuried, Germany; koch@mikrogen.de (I.K.); \\ soutschek@mikrogen.de (E.S.); boecher@mikrogen.de (O.B.) \\ 7 4th Department of Obstetrics and Gynecology, Aristotle University of Thessaloniki, Hippokratio General \\ Hospital, 54642 Thessaloniki, Greece; athetse@gmail.com (A.T.); agorast@auth.gr (T.A.) \\ 8 Lab of Computing and Medical Informatics, Department of Medicine, Aristotle University of Thessaloniki, \\ 54124 Thessaloniki, Greece; nicmag@med.auth.gr \\ 9 Research Institute for Biomedical Aging Research, University of Innsbruck, \\ A-6020 Innsbruck, Austria; pidder.jansen-duerr@uibk.ac.at \\ * Correspondence: kimon.chatzistamatiou@gmail.com; Tel.: +30-6973-321162
}

Received: 5 January 2018; Accepted: 29 January 2018; Published: 31 January 2018

\begin{abstract}
Persistent cervical infection with high-risk human papillomaviruses (hrHPVs) is a necessary, but not sufficient, condition for the development of cervical cancer. Therefore, there are other co-factors facilitating the hrHPV carcinogenic process, one of which is smoking. To assess the effect of smoking on high-risk (hr) HPV DNA positivity and on the expression of HPV E7 oncoprotein, as a surrogate of persistent hrHPV infection, we used data from women recruited for the PIPAVIR project, which examined the role of E7 protein detection in cervical cancer screening. Women were tested for hrHPV DNA, using Multiplex Genotyping (MPG), and E7 protein, using a novel sandwich ELISA method, and gave information on their smoking habits. Among 1473 women, hrHPV prevalence was $19.1 \%$. The odds ratio (OR) for hrHPV positivity of smokers compared to non-smokers was 1.785 (95\% confidence intervals (CI): 1.365-2.332, $p<0.001$ ). The ORs for E7 positivity, concerning hrHPV positive women, ranged from 0.720 to 1.360 depending on the E7 detection assay used, but this was not statistically significant. Smoking increases the probability of hrHPV infection, and smoking intensity is positively associated to this increase. Smoking is not related to an increased probability of E7 protein positivity for hrHPV positive women.
\end{abstract}

Keywords: high-risk HPV infection; E7 oncoprotein; cigarette smoking; HPV carcinogenesis; cervical cancer 


\section{Introduction}

Persistent cervical infection with high-risk human papillomaviruses (hrHPVs) is a necessary condition for the development of cervical cancer since it has been shown that virtually all cases of cervical cancer are related to HPV DNA detection [1]. However, other co-factors seem to play a role in cervical cancer development, either by facilitating hrHPV transmission and infection or by accelerating the carcinogenic process leading to cervical cancer [2]. The most important factors identified as co-factors to cervical carcinogenesis in hrHPV positive women are high parity, long-term oral contraceptive (OC) use, smoking, and co-infection with other sexually transmitted agents [3]. Particularly, parity and smoking seem to be more consistently regarded as factors modulating the risk of progression from HPV infection to cervical precancer and cancer [4].

Smoking has been shown to be a factor associated to a higher probability of hrHPV infection by studies based on hrHPV DNA detection [5-7]. However, persistent hrHPV infection, characterized by the expression of E6 and E7 oncoproteins, which play a crucial role in HPV-related carcinogenesis [8], has not yet been studied thoroughly concerning probable associations to smoking.

The present analysis aims to assess the effect of smoking on the expression of HPV E7 oncoprotein, as a surrogate of persistent hrHPV infection, and therefore to investigate whether smoking is a factor involved in the progression of hrHPV infection to cervical precancer and cancer in the clinical setting of the PIPAVIR (detection of persistent infections by human papillomaviruses) study, a study designed for the development and initial clinical assessment of a novel HPV E7 detection method for cervical cancer screening. According to the PIPAVIR study, hrHPV cervical infection was assessed using a PCR (polymerace chain reaction)-based HPV DNA detection method and five different sandwich ELISA assays identifying the E7 protein of different combinations of hrHPV types.

\section{Results}

\subsection{Demographic Data}

Demographic characteristics of the study population have been described previously [9]. Briefly, the current analysis was conducted on 1473 women aged 30-60 years old who were enrolled for the PIPAVIR project and for whom there was a valid result for hrHPV DNA genotyping and E7 protein detection. Among these women, $776(52.7 \%)$ were non-smokers, 605 (41.1\%) were active smokers and there was a smaller group of 92 women $(6.2 \%)$ who were ex-smokers as defined in the methods section. Among smokers, 181 women had a SII (Smoking intensity Index) $\leq 50,161$ women had $50<\mathrm{SII} \leq 100$, and 251 women had a SII $>100$. High-risk HPV prevalence for the study population was $19.1 \%$ (282 women found positive for hrHPV out of 1473) (Table 1).

Table 1. Demographic characteristics of the study population.

\begin{tabular}{lc}
\hline Demographic Characteristic & $\boldsymbol{n}=\mathbf{1 4 7 3}$ \\
\hline Age (years) & \\
Mean (sd) & $43.52(8.05)$ \\
Median (range) & $44(30-60)$ \\
\hline Children & \\
No & $314(21.3 \%)$ \\
Yes & $1159(78.7 \%)$ \\
\hline Smoking History & \\
No & $776(52.7 \%)$ \\
Yes & $605(41.1 \%)$ \\
Ex-Smoker & $92(6.2 \%)$ \\
\hline
\end{tabular}


Table 1. Cont.

\begin{tabular}{lc}
\hline Demographic Characteristic & $\boldsymbol{n = 1 4 7 3}$ \\
\hline SII & 1369 \\
$=0$ & 776 \\
$<50$ & 181 \\
$50-100$ & 161 \\
$>100$ & 251 \\
\hline Pap Test (at least once) & $28(1.62 \%)$ \\
No & $1695(98.37 \%)$ \\
Yes & \\
\hline hrHPV & $1191(80.9 \%)$ \\
Negative & $282(19.1 \%)$ \\
Positive &
\end{tabular}

hrHPV: high-risk human papillomavirus; SII: smoking intensity index (referring to the product of the number of cigarettes smoked per day by the years of smoking by 365 divided by 1000).

\subsection{Sample Stratification According to Smoking and Age}

We considered different groups of women regarding their smoking status (smokers, ex-smokers and non-smokers) and performed the analysis according to three distinct scenarios: (a) all three categories are independent; (b) the ex-smokers and smoker groups are merged; and (c) the ex-smokers and non-smoker groups are merged.

SII was used both as a continuous variable and as a categorical one. In particular, women were assigned to four groups, with group " 1 " consisting of non-smokers, while smokers were distributed into three groups (2,3, and 4) with SII values less than 50, between 50 and 100, and over 100, respectively. We assessed separate age categories, but the analysis did not yield significant findings and differences between these categories.

\subsection{Smoking and hrHPV DNA Positivity}

The analysis was initially focused on the high-risk HPV DNA detection, resulting to odds ratios suggesting that smoking is, indeed, significantly related to testing positive for hrHPV. As shown in Table 2, in Scenario (a), the odds ratio of smokers compared to the reference category (non-smokers) was 1.785 (95\% confidence intervals (CI): 1.365-2.332, $p<0.001$ ) indicating that the odds of being hrHPV positive were 1.785 times higher for smokers compared to non-smokers. The same has been confirmed in Scenarios (b) and (c), for which the incorporation of ex-smokers to the smoking and non-smoking group has resulted in OR > 1, 1.626 (95\% CI: 1.251-2.113, $p<0.001$ ) and 1.838 (95\% CI: 1.415-2.388, $p<0.001)$, respectively, indicating statistically significantly higher odds of being hrHPV positive in the smoking group for both scenarios. The comparison of ex-smokers to non-smokers yielded a not statistically significant difference (OR $=0.735,95 \%$ CI: $0.380-1.421, p=0.360)$ in Scenario (a). The ORs at subsequent analysis were also adjusted for the number of pregnancies and the use of oral contraceptives yielding non-significant differences compared to the unadjusted ORs. 
Table 2. Association between smoking and high-risk human papillomavirus status. The cross-tabulation tables are displayed per horizontal panel along with the odds ratios $(95 \% \mathrm{CI})$ and the corresponding $p$-values (binary logistic regression).

\begin{tabular}{|c|c|c|c|c|c|c|c|}
\hline & hrHPV (-) & hrHPV (+) & Total & OR (95\% CI) & $p$-Value & Adjusted OR * $(95 \% \mathrm{CI})$ & $p$-Value \\
\hline \multicolumn{8}{|l|}{ Scenario (a) } \\
\hline Non-Smokers (reference) & $655(84.4 \%)$ & $121(15.6 \%)$ & $776(100 \%)$ & & & & \\
\hline Smokers & $455(75.2 \%)$ & $150(24.8 \%)$ & $605(100 \%)$ & $1.785(1.365-2.332)$ & $<0.001$ & $1.788(1.367-2.340)$ & $<0.001$ \\
\hline Ex-Smokers & $81(88 \%)$ & $11(12 \%)$ & $92(100 \%)$ & $0.735(0.380-1.421)$ & 0.360 & $0.682(0.350-1.329)$ & 0.261 \\
\hline Total & $1191(80.9 \%)$ & $282(19.1 \%)$ & $1473(100 \%)$ & & & & \\
\hline \multicolumn{8}{|l|}{ Scenario (b) } \\
\hline Non-Smokers (reference) & $655(84.4 \%)$ & $121(15.6 \%)$ & $776(100 \%)$ & & & & \\
\hline Smokers and ex-smokers & $536(76.9 \%)$ & $161(23.1 \%)$ & $697(100 \%)$ & $1.626(1.251-2.113)$ & $<0.001$ & $1.618(1.244-2.106)$ & $<0.001$ \\
\hline Total & $1191(80.9 \%)$ & $282(19.1 \%)$ & $1473(100 \%)$ & & & & \\
\hline \multicolumn{8}{|l|}{ Scenario (c) } \\
\hline Non- and ex-smokers (reference) & $736(84.8 \%)$ & $132(15.2 \%)$ & $868(100 \%)$ & & & & \\
\hline Smokers & $455(75.2 \%)$ & $150(24.8 \%)$ & $605(100 \%)$ & $1.838(1.415-2.388)$ & $<0.001$ & $1.856(1.427-2.413)$ & $<0.001$ \\
\hline Total & $1191(80.9 \%)$ & $282(19.1 \%)$ & $1473(100 \%)$ & & & & \\
\hline \multicolumn{8}{|l|}{ Smoking Intensity Index } \\
\hline SII $=0$ (reference) & $655(84.4 \%)$ & $121(15.6 \%)$ & $776(100 \%)$ & & & & \\
\hline $0<\mathrm{SII}<50$ & $133(73.5 \%)$ & $48(26.5 \%)$ & $181(100 \%)$ & $1.954(1.332-2.865)$ & 0.001 & $1.828(1.242-2.690)$ & 0.002 \\
\hline $50<$ SII $<100$ & $112(69.6 \%)$ & $49(30.4 \%)$ & $161(100 \%)$ & $2.368(1.607-3.490)$ & $<0.001$ & $2.272(1.538-3.356)$ & $<0.001$ \\
\hline $\mathrm{SII}>100$ & $201(80.1 \%)$ & $50(19.9 \%)$ & $251(100 \%)$ & $1.347(0.934-1.941)$ & 0.111 & $1.471(1.015-2.131)$ & 0.041 \\
\hline Total & $1101(80.4 \%)$ & $268(19.6 \%)$ & $1369(100 \%)$ & & & & \\
\hline
\end{tabular}

hrHPV: High-Risk Human Papillomavirus; SII: Smoking Intensity Index; OR: Odds Ratio; ${ }^{*}$ adjustment was performed for the number of pregnancies and the use of oral contraceptives. 
Concerning SII, it was shown that women who were smokers had higher odds to be positive for hrHPV compared to non-smokers, regardless of them being light, medium or heavy smokers. In detail, women with SII below 50 presented OR of 1.954 (95\% CI: 1.332-2.865, $p=0.001$ ), and women with SII between 50 and 100 presented higher OR (2.368 (95\% CI: 1.607-3.490, $p<0.001)$ ), which means that women who had smoked more were more probable to be hrHPV positive compared to non-smokers than those who were light smokers. However, interestingly, the same result was not observed concerning heavy smokers, since they presented OR (unadjusted) of 1.347 (95\% CI: 0.934-1.941, $p=0.111$ ) of being hrHPV positive, a value which did not reveal a statistically significant difference compared to non-smokers as one would expect (Table 2). However, statistical significance was obtained after adjustment, which yielded a OR of 1.471 (95\% CI: 1.015-2.131, $p=0.041$ ).

\subsection{Smoking and E7 Detection}

Regarding the results for the five different E7-testing assays, labeled recomWell HPV 16/18/45 $\mathrm{KJ}_{\text {high }}$, recomWell HPV 39/51/56/59, recomWell HPV 16/31/33/35/52/58, recomWell HPV HR screen and recomWell HPV $16 / 18 / 45 \mathrm{KJ}_{\text {low }}$, only women who had previously given a hrHPV positive result were selected, namely 282 women (19.1\%). Among them, 121 (42.9\%) were non-smokers, $150(53.2 \%)$ were current smokers and $11(3.9 \%)$ were ex-smokers (Table 3$)$.

Table 3. Frequency table regarding smoking status for hrHPV positive women.

\begin{tabular}{ccc}
\hline & Frequency $(\boldsymbol{n})$ & Percentage (\%) \\
\hline Non-Smokers & 121 & 42.9 \\
Smokers & 150 & 53.2 \\
Ex-Smokers & 11 & 3.9 \\
Total & 282 & 100.0 \\
\hline Smoking Intensity & \\
Index & & \\
SII = 0 & 121 & 45.1 \\
$0<$ SII $<50$ & 48 & 17.9 \\
$50<$ SII $<100$ & 49 & 18.3 \\
SII $>100$ & 50 & 18.7 \\
\hline \multicolumn{2}{c}{ SII. Smoking Intensity Index }
\end{tabular}

SII: Smoking Intensity Index.

This analysis involved the three scenarios as previously described and yielded no statistically significant differences concerning E7 positivity for hrHPV positive women who were smokers compared to non-smokers in either of the three (Table 4). The most important finding was the higher than 1 ORs, presented by recomWell HPV 39/51/56/59 assay, in all three scenarios examined, meaning that smokers who were hrHPV positive tended to have an increased possibility for positive E7 testing compared to non-smokers, however none of these results were statistically significant and, therefore, this correlation could not be established. Adjustment did not reveal statistically significant differences for any of the E7 tests examined and the ORs were only changed slightly. 
Table 4. Association between smoking/smoking intensity and E7 for hrHPV positive women. The odds ratios (95\% CI) and the corresponding $p$-values (binary logistic regression) are displayed per horizontal panel. Cross-tabulation tables are not displayed due to space limitation.

\begin{tabular}{|c|c|c|c|c|c|}
\hline \multirow[t]{2}{*}{ E7 Detection Assays } & $\begin{array}{l}\text { recomWell HPV } \\
16 / 18 / 45 \mathrm{KJ}_{\text {high }}\end{array}$ & $\begin{array}{c}\text { recomWell HPV } \\
39 / 51 / 56 / 59\end{array}$ & $\begin{array}{l}\text { recomWell HPV } \\
16 / 31 / 33 / 35 / 52 / 58\end{array}$ & $\begin{array}{c}\text { recomWell HPV HR } \\
\text { Screen }\end{array}$ & $\begin{array}{l}\text { recomWell HPV } \\
16 / 18 / 45 \mathrm{KJ}_{\text {low }}\end{array}$ \\
\hline & OR ( $p$-Value) $95 \%$ CI & OR ( $p$-Value) $95 \%$ CI & OR ( $p$-Value) $95 \%$ CI & OR ( $p$-Value) $95 \%$ CI & OR ( $p$-Value) $95 \%$ CI \\
\hline \multicolumn{6}{|l|}{ Scenario (a) } \\
\hline \multicolumn{6}{|l|}{ Non-Smokers (reference) } \\
\hline Smokers & $0.720(0.182) 0.444-1.167$ & $1.360(0.233) 0.820-2.256$ & 0.907 (0.707) $0.545-1.510$ & $0.966(0.889) 0.591-1.578$ & $0.838(0.492) 0.506-1.388$ \\
\hline Ex-Smokers & $1.971(0.334) 0.498-7.798$ & $1.798(0.357) 0.516-6.261$ & $1.101(0.883) 0.305-3.980$ & $0.773(0.684) 0.223-2.676$ & $0.987(0.984) 0.274-3.562$ \\
\hline \multicolumn{6}{|l|}{ Scenario (b) } \\
\hline \multicolumn{4}{|l|}{ Non-Smokers (reference) } & $0.951(0.838) 0.587-1.542$ & $0.848(0.514) 0.516-1.392$ \\
\hline \multicolumn{6}{|l|}{ Scenario (c) } \\
\hline \multicolumn{6}{|l|}{ Non- and ex-Smokers (reference) } \\
\hline Smokers & $0.683(0.114) 0.426-1.096$ & $1.290(0.309) 0.790-2.108$ & 0.899 (0.676) 0.547-1.479 & $0.987(0.958) 0.612-1.594$ & $0.839(0.485) 0.513-1.373$ \\
\hline Smoking Intensity Index (SII) & $0.996(0.093) 0.992-1.001$ & 1.000 (0.902) 0.995-1.004 & 0.999 (0.588) $0.994-1.003$ & $0.997(0.135) 0.992-1.001$ & 0.999 (0.688) 0.995-1.004 \\
\hline \multicolumn{6}{|l|}{$\mathrm{SII}=0$ (reference) } \\
\hline $0<\mathrm{SII}<50$ & 1.035 (0.921) 0.525-2.039 & $1.414(0.329) 0.706-2.832$ & $0.963(0.918) 0.474-1.957$ & $0.901(0.879) 0.456-1.781$ & 0.947 (0.879) 0.471-1.904 \\
\hline \%cmidrule1-6 $50<$ SII $<100$ & $0.710(0.313) 0.364-1.382$ & $1.758(0.105) 0.889-3.477$ & $1.119(0.715) 0.560-2.236$ & $1212(0.993) 0.606-2.424$ & 1.003 (0.993) $0.503-1.998$ \\
\hline $\mathrm{SII}>100$ & $0.581(0.109) 0.299-1.130$ & $0.925(0.831) 0.452-1.894$ & $0.749(0.434) 0.363-1.545$ & $0.819(0.182) 0.420-1.598$ & 0.607 (0.182) 0.292-1.263 \\
\hline
\end{tabular}

SII: Smoking Intensity Index; OR: Odds Ratio. 


\section{Discussion}

According to the International Agency for Research on Cancer (IARC) certain types of human papillomaviruses are considered carcinogenic to humans. These types are HPV 16, the most potent carcinogen, and others (HPVs 18, 31, 33, 35, 39, 45, 51, 52, 56, 58, and 59) for which there is sufficient evidence concerning carcinogenicity. Other types are also thought to have a carcinogenic potential for which there is limited evidence [10]. The natural history of hrHPV infection, however, dictates that only a minority of cervical hrHPV infections will eventually lead to cervical cancer [11]. On the other hand, virtually all cases of cervical cancer are related to a hrHPV infection [1]. Therefore, according to these facts, hrHPV infection is considered a necessary but not sufficient condition for cervical carcinogenesis, which is facilitated by certain known or unknown co-factors [4].

Smoking is a known co-factor for hrHPV related carcinogenesis. In general, women who smoke are more frequently found hrHPV positive compared to non-smokers according to a study conducted in Greece [5], the main site of the presented study too, as well as according to other studies in different countries [6,12-15]. Moreover, the intensity of smoking seems to play a role in the risk of being hrHPV positive, as has been shown by a pooled IARC analysis [16], and by the Greek study too [5]. The former analysis considered women who smoked at least one cigarette per day for at least one year as smokers and the latter used the SII, a variable introduced by our group then, and used in the current analysis too, which is strongly influenced by the time during which a woman has been a smoker. This is an advantage of that index which may allow for more robust results. The current analysis has shown a higher probability for women who currently smoke compared to non-smokers and an even higher probability for women who smoke more. However, as already noted, women who have an SII > 100 (heavy smokers) do not differ statistically significantly compared to non-smokers in the unadjusted model, but they do differ statistically significantly in the adjusted model. Although this is a rather unexpected result, it could be justified, since the effect of smoking on HPV testing does not necessarily exhibit a monotone relation, and, moreover, the thresholds 50 and 100 used to define the categories are arbitrary.

A third group of women, comprised of ex-smokers, was also identified which involved women who had stopped smoking at least a year ago, according to previous research practices [16]. These women were only $92 / 1473(6.2 \%)$ in the current analysis, however, since they are a special group, they were analyzed as such (Scenario (a)) and also as being either smokers (Scenario (b)) or non-smokers (Scenario (c)). Ex-smokers and non-smokers did not exhibit any statistically significant differences in the odds for being hrHPV positive. Furthermore, when ex-smokers were grouped with smokers, the OR of being hrHPV positive was decreased compared to the one deriving from them being grouped with non-smokers (1.626 vs. 1.838). These results might suggest the rapid effect of the protective role of smoking cessation concerning hrHPV infection.

The molecular mechanism of hrHPV induced carcinogenesis is based on the crucial role of E6 and E7 viral oncoproteins which are involved in the degradation of p53 [17-19] and reduced activity of pRB (Retinoblastoma protein) [20,21], respectively. The current analysis, apart from examining the role of smoking on the probability of hrHPV cervical infection, mainly aimed to assess its role on the progression of hrHPV infection from a transient to a productive one, identified by the detection of E7 protein, the most potent factor in HPV related carcinogenesis. To our knowledge, this is being investigated for the first time, and, interestingly, it was shown that smoking did not increase the probability of E7 positivity, as was determined using the novel E7 assays developed for clinical use during the PIPAVIR project. The target group for this analysis had been women of the PIPAVIR cohort, tested positive for hrHPV DNA using a PCR-based method, which implies the detection of hrHPV DNA but does not give information on the occurrence of transient or transforming hrHPV infection.

The modifying role of smoking in the natural history of hrHPV infection is not yet clear and epidemiologic studies often reach controversial results, probably due to methodologic issues which produce bias, since elimination of confounding is not always possible [22]. It seems that smoking interferes with the immune system by suppressing it [23] and this mechanism has been proposed as the 
main link between smoking and hrHPV infection. Recently, the first relevant study to investigate by complex modeling indirect (antibody-mediated) and direct (antibody-independent) effects of smoking on HPV infection showed that current smokers had 29\% increased odds for HPV16 infection by the indirect mechanism, an effect which was statistically significant, whereas this was not shown for the direct effect of smoking [24]. Moreover, the indirect effect of smoking was more intense $(61 \%$ increased odds) in women who smoked more per day, but a significant increase was not observed concerning smoking duration. Former smokers were also not reported to express a significant antibody-mediated effect on HPV16 infection. These findings describe a mechanism which could explain the results of the current analysis, namely the higher probability, and the insignificantly different probability of hrHPV infection in current and former smokers, respectively, compared to never-smokers. An immune mechanism behind the effect of smoking on hrHPV infection would most probably imply an effect early in the natural history of the infection, by preventing the virus-host cell membrane interaction, and not on later stages characterized by expression of E6/E7 viral proteins. In our sub-analysis, the non-significant effect of smoking on the detection of E7 protein, an indicator of persistent hrHPV infection in vivo, supports further the suggestion, that cigarette smoking has a more prominent role in earlier stages of HPV-related carcinogenesis. However, more research is needed to clarify the molecular mechanisms by which smoking interacts with the natural history of hrHPV infection.

As a conclusion, smoking seems to increase the probability of hrHPV infection, and smoking intensity is positively associated to this increase. However, smoking is not related to an increased probability of E7 protein positivity concerning hrHPV positive women.

\section{Materials and Methods}

\subsection{Study Design and Sampling}

The PIPAVIR project was a study conducted between August 2012 and August 2015, aiming at the development and initial clinical assessment of a novel ELISA hrHPVE7 oncoprotein detection method for cervical cancer screening. The study design has been described in detail previously [9]. Briefly, participants were non-pregnant women aged 30-60, without a history of cervical intraepithelial neoplasia (CIN), who visited the Family Planning Centre, Hippokratio Hospital of Thessaloniki, Greece and the Department of Gynecology and Obstetrics in Im Mare Klinikum, Kiel, Germany and gave their written informed consent to participate. Subsequently, a cervicovaginal sample, used for Thinprep cytology, HPV DNA genotyping and hrHPVE7 protein detection was taken and women with a positive cytology or hrHPV DNA result were referred to colposcopy followed by biopsy and/or endocervical curettage (ECC), when needed. Sampling was performed using the CervexBrush (Rovers Medical Devices, Lekstraat 10, NL-5347, KV Oss, The Netherlands) and the Cytobrush (CooperSurgical, Inc., 95 Corporate Drive, Trumbull, CT, USA) according to the manufacturer's instructions. After sampling both brushes were immersed in a vial containing collection fluid (PreservCyt, Hologic, Bedford, MA, USA).

\subsection{Smoking Habits Assessment}

A personal information sheet was filled in for each woman enrolled in the study including demographic characteristics. Concerning smoking, each woman was asked to identify herself as a current smoker, a non-smoker (never smoker), or an ex-smoker, meaning that she had quit smoking at least one year prior to recruitment. Smokers were also asked for more specific information about their smoking habits, in particular, about the number of cigarettes smoked per day as well as their smoking years. This information allowed a more quantitative approach through Smoking Intensity Index (SII), a new variable created by the number of cigarettes smoked per day multiplied by 365 and by years of smoking and divided by 1000 ( $n$ (cigarette/day) $\times 365 \times n$ (years) $\div 1000$ ) [5]. In this way, women were divided into light smokers (SII < 50), medium smokers (SII 50-100) and heavy smokers (SII > 100). 


\subsection{HPV DNA Genotyping}

HPV genotyping by Multiplexed Genotyping (MPG), consisting of a consensus broad-spectrum GP5+/GP6+ primer multiplex PCR and a type-specific probe read out by Luminex technology was performed at the CHARITE, Gynecologic Tumor Immunology Laboratory, Berlin, Germany [25,26]. MPG, targets the L1 gene sequence, and identifies hrHPV types 16, 18, 26, 31, 33, 35, 39, 45, 51, 52, 53, $56,58,59,66,68,73$, and 82, and low-risk HPVs 6, 11, 42, 54, 70, 72 and 90.

\section{4. hrHPVE7 Testing}

A sandwich ELISA method was developed during the PIPAVIR project to detect hrHPVE7 protein, as a result of a collaboration between the laboratories of Innsbruck University and Mikrogen GmbH, Neuried, Germany [9]. The detection strategy for hrHPVE7 was based on five different assays: "recomWell HPV 16/18/45 KJhigh", "recomWell HPV 16/18/45 KJlow", "recomWell HPV 39/51/56/59", "recomWell HPV 16/31/33/35/52/58" and "recomWell HPV HR screen" (for 16, 18, $31,33,35,39,45,51,52,56,58$ and 59 E7). Each assay refers to different combinations of hrHPVE7 or different concentrations of the relevant ELISA antibodies and yields a measurement of the optical density, a continuous variable.

\subsection{Statistical Analysis}

The main purpose of the analysis was to validate the association between HPV infection, smoking and smoking intensity, and to assess for the first time the association between E7 protein detection, smoking and smoking intensity for hrHPV positive women. The result of the HPV test was a categorical binary variable, whereas the optical density value derived from the measurement of the E7 oncoprotein was a continuous numerical variable. For this analysis, this continuous variable was transformed into a binary one, by a cut-off value calculated using receiver operating characteristic curve analysis. The impact of smoking and smoking intensity on hrHPV infection was evaluated empirically by cross-tabulation matrices (in the case of E7, cross-tabulation matrices are not displayed due to space limitation). The binary logistic regression was selected to statistically evaluate the association between $\mathrm{HPV} / \mathrm{E} 7$ and smoking/smoking intensity, since it was of interest to investigate three categories in terms of smoking (smokers, ex-smokers and non-smokers) and four categories in terms of smoking intensity (SII $=0,0<\mathrm{SII} \leq 50,50<\mathrm{SII} \leq 100$ and SII $>100$ ), and their binary correlations (one category was selected each time as a reference category). The selected regression model was applied unadjusted to assess smoking/smoking intensity, and adjusted for the number of pregnancies and the combined oral contraceptive use (COC). The odds ratios (ORs) and adjusted ORs along with their $95 \%$ confidence intervals (CI) and the corresponding p-values were computed. The significance level was set to 0.05 . $p$-value $<0.05$ indicated statistically significantly different ORs compared to one. All analyses were performed using the statistical software SPSS Statistics 22.0.

\subsection{Ethical Approval}

Allproceduresperformed in studies involving human participants were in accordance with the ethical standards of the institutional and/or national research committee and with the 1964 Helsinki declaration and its later amendments or comparable ethical standards. The study was performed subject to the: Bioethics Committee of the Medical School of Aristotle University of Thessaloniki/Greece (33-31/8/2012) and the Ethikkommission der CHARITE-Universitätsmedizin Berlin/Germany (Available online: http:/ / www.charite.de/fakultaet/ kommissionen/ethikkommission.html). Project identification date: 31 August 2012.

\subsection{Informed Consent}

Informed consent was obtained from all individual participants included in the study. 
Acknowledgments: The authors acknowledge the contribution of the following colleagues and collaborators: Achim Schneider, Lutz Gissmann, and Silvia de Sanjose for their invaluable advice during the project; Ursula Schiller for expert technical assistance with Multiplex Genotyping; Garifallia Michalaki and Georgia Kiriafini for nursing assistance during sampling; Thomas Theodoridis ${ }^{\dagger}$ and Fausto Carcea for clinical supervision during the sampling procedure; Anastasia Kitsou for administrative work; and The Mikrogen team (Mira Kellner, Stefanie Fehrmann, Mandy Fleischhauer, Steven McNamara, Melanie Thiessen, and Sophie Vetter) for technical assistance with E7 ELISA test development and measurements of the clinical samples. The trial was funded by the EU 7th framework programme, FP7-HEALTH-304927. $\left({ }^{\dagger}\right.$ : deceased).

Author Contributions: Theodoros Agorastos and Kimon Chatzistamatiou conceived and designed the study, wrote the manuscript and supervised statistical analysis; Theodoros Moysiadis and Dimos Vryzas performed the statistical analysis and wrote parts of the manuscript; Athena Tsertanidou handled the database and drafted parts of the manuscript; Ekaterini Chatzaki supervised statistical analysis and reviewed the manuscript; Andreas M. Kaufmann performed and supervised all hrHPV DNA testing and wrote parts of the manuscript; Isabel Koch, Erwin Soutschek and Oliver Boecher participated in the E7 testing development, performed and supervised all E7 testing and wrote parts of the manuscript; Nikolaos Maglaveras supervised software design for the data capturing system used for the study and reviewed the manuscript; and Pidder Jansen-Duerr developed the E7 testing method and reviewed the manuscript.

Conflicts of Interest: Kimon Chatzistamatiou and Theodoros Agorastos have had travel expenses and fees for congresses covered by Roche Diagnostics and SPMSD (Sanofi Pasteur MSD). Theodoros Agorastos has conducted studies partially supported by Vianex S.A and SPMSD, and has received research grants by Volkswagen, Bodossakis and Papageorgiou foundations. Pidder Jansen-Duerr is listed as an inventor on a patent application describing the use of HPV E7 antibodies. Isabel Koch, Oliver Boecher and Erwin Soutschek are employees of MIKROGEN. All other authors report no conflict of interest concerning the submitted article. The authors have had full control of all primary data and agree to allow the Journal to review their data if requested.

\author{
Abbreviations \\ hrHPV High-Risk Human Papillomavirus \\ DNA Directory Deoxyribonucleic Acid \\ ELISA Enzyme-Linked Immune Sorbent Assay \\ IARC International Agency for the Research on Cancer \\ OR Odds Ratio \\ pRB Retinoblastoma Protein \\ PCR Polymerace Chain Reaction \\ MPG Multiplex Genotyping \\ CIN Cervical Intraepithelial Neoplasia
}

\title{
References
}

1. Walboomers, J.M.M.; Jacobs, M.V.; Manos, M.M.; Bosch, F.X.; Kummer, J.A.; Shah, K.V.; Snijders, P.J.F.; Peto, J.; Meijer, C.J.L.M.; Munoz, N. Human papillomavirus is a necessary cause of invasive cervical cancer worldwide. J. Pathol. 1999, 189, 12-19. [CrossRef]

2. Arends, M.J.; Buckley, C.H.; Wells, M. Aetiology, pathogenesis, and pathology of cervical neoplasia. J. Clin. Pathol. 1998, 51, 96-103. [CrossRef] [PubMed]

3. Castellsague, X.; Bosch, F.X.; Munoz, N. Environmental co-factors in HPV carcinogenesis. Virus. Res. 2002, 89, 191-199. [CrossRef]

4. Castellsague, X.; Munoz, N. Chapter 3: Cofactors in human papillomavirus carcinogenesis—Role of parity, oral contraceptives, and tobacco smoking. J. Natl. Cancer Inst. Monogr. 2003, 2003, 20-28. [CrossRef]

5. Chatzistamatiou, K.; Katsamagas, T.; Zafrakas, M.; Zachou, K.; Orologa, A.; Fitsiou, F.; Theodoridis, T.; Konstantinidis, T.; Konstantinidis, T.C.; Agorastos, T. Smoking and genital human papilloma virus infection in women attending cervical cancer screening in greece. World J. Obstet. Gynecol. 2013, 2, 53. [CrossRef]

6. Pista, A.; de Oliveira, C.F.; Cunha, M.J.; Paixao, M.T.; Real, O.; Group, C.P.S. Risk factors for human papillomavirus infection among women in portugal: The cleopatre portugal study. Int. J. Gynaecol. Obstet. 2012, 118, 112-116. [CrossRef] [PubMed]

7. Vaccarella, S.; Franceschi, S.; Herrero, R.; Munoz, N.; Snijders, P.J.; Clifford, G.M.; Smith, J.S.; Lazcano-Ponce, E.; Sukvirach, S.; Shin, H.R.; et al. Sexual behavior, condom use, and human papillomavirus: Pooled analysis of the iarc human papillomavirus prevalence surveys. Cancer Epidemiol. Biomark. Prev. 2006, 15, 326-333. [CrossRef] [PubMed] 
8. Narisawa-Saito, M.; Kiyono, T. Basic mechanisms of high-risk human papillomavirus-induced carcinogenesis: Roles of E6 and E7 proteins. Cancer Sci. 2007, 98, 1505-1511. [CrossRef] [PubMed]

9. Agorastos, T.; Chatzistamatiou, K.; Moysiadis, T.; Kaufmann, A.M.; Skenderi, A.; Lekka, I.; Koch, I.; Soutschek, E.; Boecher, O.; Kilintzis, V.; et al. Human papillomavirus E7 protein detection as a method of triage to colposcopy of hpv positive women, in comparison to genotyping and cytology. Final results of the pipavir study. Int. J. Cancer 2017, 141, 519-530. [CrossRef] [PubMed]

10. Bouvard, V.; Baan, R.; Straif, K.; Grosse, Y.; Secretan, B.; El Ghissassi, F.; Benbrahim-Tallaa, L.; Guha, N.; Freeman, C.; Galichet, L.; et al. A review of human carcinogens-Part b: Biological agents. Lancet Oncol. 2009, 10, 321-322. [CrossRef]

11. Schiffman, M.; Castle, P.E.; Jeronimo, J.; Rodriguez, A.C.; Wacholder, S. Human papillomavirus and cervical cancer. Lancet 2007, 370, 890-907. [CrossRef]

12. Herrero, R.; Castle, P.E.; Schiffman, M.; Bratti, M.C.; Hildesheim, A.; Morales, J.; Alfaro, M.; Sherman, M.E.; Wacholder, S.; Chen, S.; et al. Epidemiologic profile of type-specific human papillomavirus infection and cervical neoplasia in guanacaste, costa rica. J. Infect. Dis. 2005, 191, 1796-1807. [CrossRef] [PubMed]

13. Remschmidt, C.; Kaufmann, A.M.; Hagemann, I.; Vartazarova, E.; Wichmann, O.; Delere, Y. Risk factors for cervical human papillomavirus infection and high-grade intraepithelial lesion in women aged 20 to 31 years in germany. Int. J. Gynecol. Cancer 2013, 23, 519-526. [CrossRef] [PubMed]

14. Roteli-Martins, C.M.; Panetta, K.; Ferreira Alves, V.A.; Coelho Siqueira, S.A.; Syrjänen, K.J.; Mauricette Derchain, S.F.C. Cigarette smoking and high-risk HPV DNA as predisposing factors for high-grade cervical intraepithelial neoplasia (cin) in young brazilian women. Acta Obstet. Gynecol. Scand. 1998, 77, 678-682. [CrossRef] [PubMed]

15. Sellors, J.W.; Mahony, J.B.; Kaczorowski, J.; Lytwyn, A.; Bangura, H.; Chong, S.; Lorincz, A.; Dalby, D.M.; Janjusevic, V.; Keller, J.L. Prevalence and predictors of human papillomavirus infection in women in ontario, canada. Survey of HPV in ontario women (show) group. CMAJ 2000, 163, 503-508. [PubMed]

16. Vaccarella, S.; Herrero, R.; Snijders, P.J.; Dai, M.; Thomas, J.O.; Hieu, N.T.; Ferreccio, C.; Matos, E.; Posso, H.; de Sanjose, S.; et al. Smoking and human papillomavirus infection: Pooled analysis of the international agency for research on cancer HPV prevalence surveys. Int. J. Epidemiol. 2008, 37, 536-546. [CrossRef] [PubMed]

17. Hiller, T.; Poppelreuther, S.; Stubenrauch, F.; Iftner, T. Comparative analysis of 19 genital human papillomavirus types with regard to p53 degradation, immortalization, phylogeny, and epidemiologic risk classification. Cancer Epidemiol. Biomark. Prev. 2006, 15, 1262-1267. [CrossRef] [PubMed]

18. Mesplede, T.; Gagnon, D.; Bergeron-Labrecque, F.; Azar, I.; Senechal, H.; Coutlee, F.; Archambault, J. P53 degradation activity, expression, and subcellular localization of E6 proteins from 29 human papillomavirus genotypes. J. Virol. 2012, 86, 94-107. [CrossRef] [PubMed]

19. Zimmermann, H.; Degenkolbe, R.; Bernard, H.U.; O'Connor, M.J. The human papillomavirus type 16 e6 oncoprotein can down-regulate p53 activity by targeting the transcriptional coactivator cbp/p300. J. Virol. 1999, 73, 6209-6219. [PubMed]

20. Fiedler, M.; Muller-Holzner, E.; Viertler, H.P.; Widschwendter, A.; Laich, A.; Pfister, G.; Spoden, G.A.; Jansen-Durr, P.; Zwerschke, W. High level HPV-16 E7 oncoprotein expression correlates with reduced prb-levels in cervical biopsies. FASEB J. 2004, 18, 1120-1122. [CrossRef] [PubMed]

21. Frolov, M.V.; Dyson, N.J. Molecular mechanisms of e2f-dependent activation and prb-mediated repression. J. Cell Sci. 2004, 117, 2173-2181. [CrossRef] [PubMed]

22. Franco, E.L.; Spence, A.R. Commentary: Smoking and human papillomavirus infection: The pursuit of credibility for an epidemiologic association. Int. J. Epidemiol. 2008, 37, 547-548. [CrossRef] [PubMed]

23. Sopori, M. Effects of cigarette smoke on the immune system. Nat. Rev. Immunol. 2002, 2, 372-377. [CrossRef] [PubMed]

24. Eldridge, R.C.; Pawlita, M.; Wilson, L.; Castle, P.E.; Waterboer, T.; Gravitt, P.E.; Schiffman, M.; Wentzensen, N. Smoking and subsequent human papillomavirus infection: A mediation analysis. Ann. Epidemiol. 2017, 27, 724-730.e721. [CrossRef] [PubMed] 
25. Schmitt, M.; Bravo, I.G.; Snijders, P.J.; Gissmann, L.; Pawlita, M.; Waterboer, T. Bead-based multiplex genotyping of human papillomaviruses. J. Clin. Microbiol. 2006, 44, 504-512. [CrossRef] [PubMed]

26. Schmitt, M.; Dondog, B.; Waterboer, T.; Pawlita, M. Homogeneous amplification of genital human alpha papillomaviruses by pcr using novel broad-spectrum GP5+ and GP6+ primers. J. Clin. Microbiol. 2008, 46, 1050-1059. [CrossRef] [PubMed] 